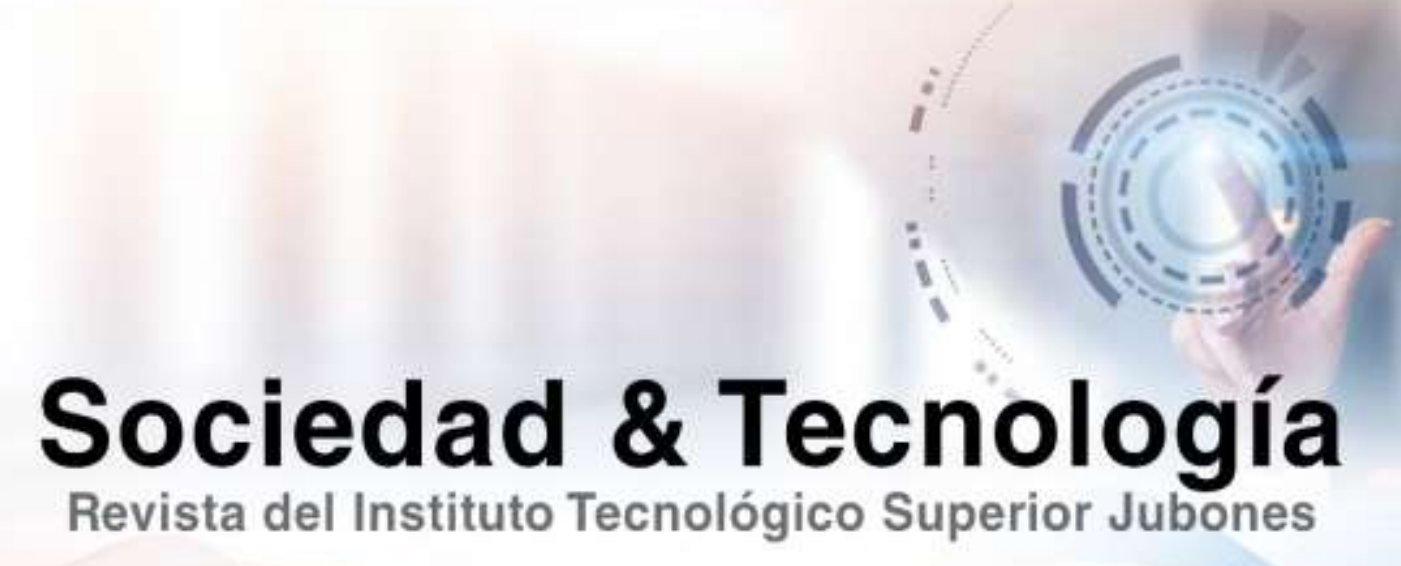

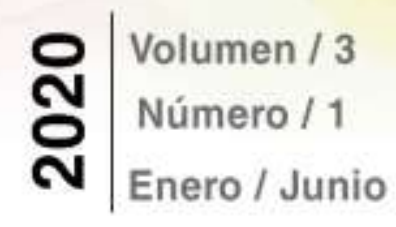

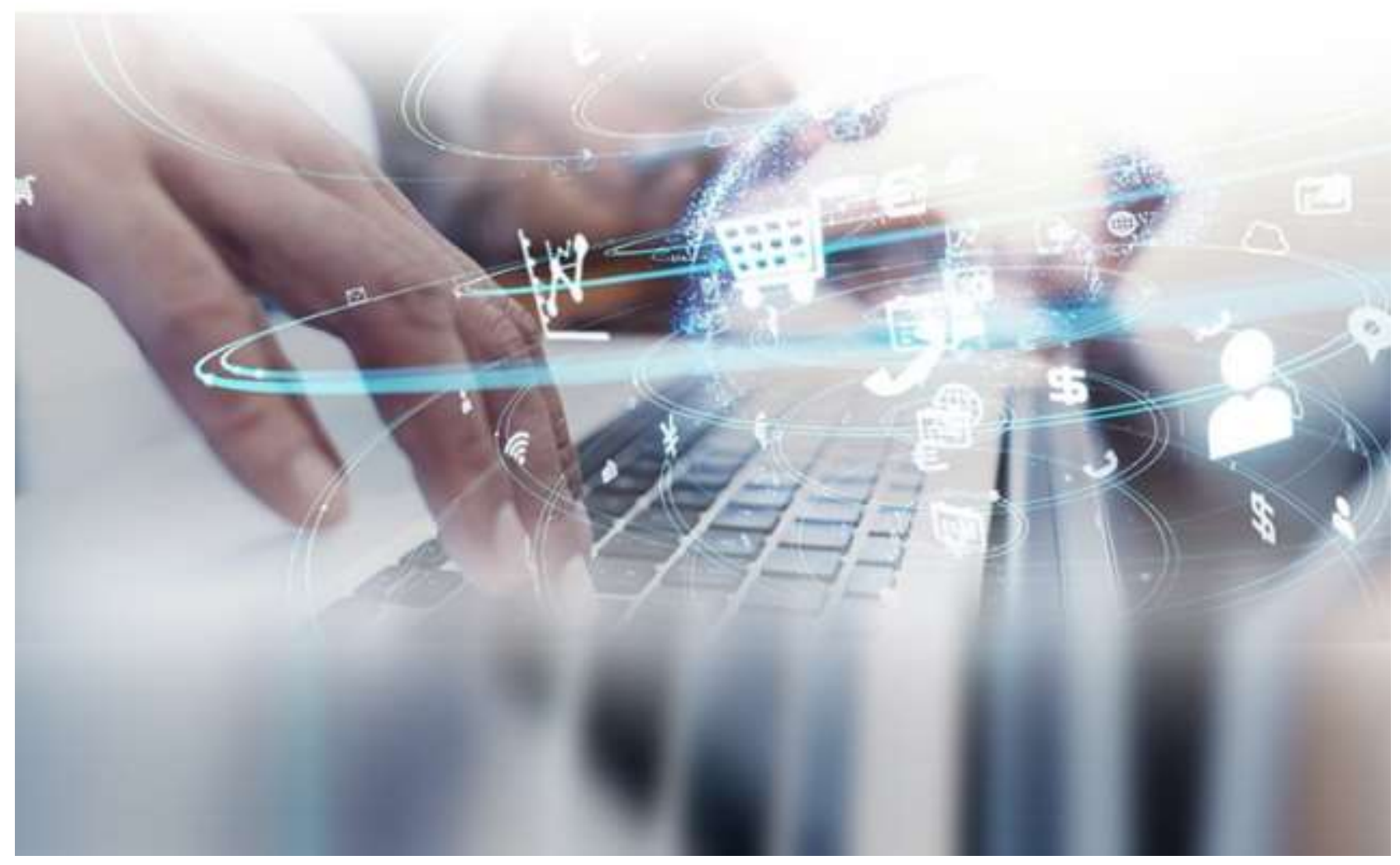




\title{
USO O ABUSO DE LAS FUENTES DE INFORMACIÓN EN LA REDACCIÓN ACADÉMICA
}

\author{
USE OR ABUSE OF INFORMATION SOURCES IN THE ACADEMIC DRAFTING
}

Leonela Yajaira Granda Asencio ${ }^{1}$

E-mail: Igranda3@utmachala.edu.ec

ORCID: https://orcid.org/0000-0001-5879-5035

Koraima Noemi Mera Machuca ${ }^{1}$

E-mail: kmera2@utmachala.edu.ec

ORCID: https://orcid.org/0000-0001-6245-9130

German Ernesto Peña Nivecela ${ }^{1}$

E-mail: gpena4@utmachala.edu.ec

ORCID: https://orcid.org/0000-0003-2870-2584

Janice Brittany Solórzano López ${ }^{1}$

E-mail: jsolorzan2@utmachala.edu.ec

ORCID: https://orcid.org/0000-0001-8577-8951

1 Universidad Técnica de Machala, Ecuador

Cita sugerida (APA, séptima edición)

Granda Asencio, L. Y., Mera Machuca, K. N., Peña Nivecela, G. E. \& Solórzano López, J. B. (2020). Uso o abuso de las fuentes de información en la redacción académica. Revista Sociedad \& Tecnología, $3(1), 1-9$.

\section{RESUMEN}

El presente ensayo tiene como objetivo el caracterizar el plagio y sus diferentes abordajes, desde una perspectiva sobre lo axiológico para la concentración de lo moral y ético en cuanto a las acciones, lo curricular por sus normar, planes y metodologías para abordarlo y finalmente lo tecnológico por su introducción a la era digital considerando beneficios y desventajas que posee, concretando perspectivas que ahondan sobre las posibles causas por las que se puede aplicar dentro del campo educativo. Se determina los contratiempos por los que pasa el individuo y consecuencias que arraiga más problemáticas donde vaya a ejercer su profesión. Además, se consideran diversos pensamientos y conceptualizaciones sobre este tópico por diferentes autores para la pertinencia del escrito, dada por medio de una recopilación bibliográfica. Obteniendo como conclusión que esta puede establecerse en diversos contextos y ser conceptualizado como una falta a la autoría, en cuanto a lo académico se transfieren otras características tales como la flexibilidad y facilidad para cometerla y en otros casos por la falta de relevancia hacia su ejecución.

\section{Palabras claves:}

educación, plagio, axiología.

\section{ABSTRACT}

The present essay aims to characterize plagiarism and its different approaches, from a perspective on the axiological for the concentration of the moral and ethical in terms of actions, the curricular for its norms, plans and methodologies to address it and finally the technological for its introduction to the digital age considering benefits and disadvantages it has, specifying perspectives that delve into the possible causes for which it can be applied within the educational field. It determines the setbacks that the individual goes through and 
the consequences that take root where they are going to practice their profession. In addition, various thoughts and conceptualizations on this topic by different authors are considered for the relevance of the writing given through a bibliographic compilation. Obtaining as a conclusion that this can be established in different contexts and be conceptualized as a lack of authorship, in terms of academic's other characteristics are transferred such as flexibility and ease to commit it and in other cases due to the lack of relevance towards its execution.

\section{Keywords:}

education, plagiarism, axiology.

\section{INTRODUCCIÓN}

El llamado plagio es considerado como una práctica anti-moral y ética, de las cuales algunos individuos con falta de principios y valores las suelen cometer innumerables errores en trabajos académicos, algunos caen por desconocimiento y muchos otros con intención. El objetivo de este ensayo es contribuir e informar las consecuencias que conlleva esta temática del plagio académico, el famoso plagio académico conocido así dentro del ámbito educativo se examina como un robo y uso ilegal de ideas, materiales y obras que poseen otros autores. El plagio ocasiona un sinnúmero de problemáticas y no hace más que limitar a una sociedad en crecimiento.

Es de bien conocer que la deshonestidad académica se denomina como una ausencia de ética y entabla acciones que deben ser sancionadas por la intencionalidad con la que se lo comete, sin dejar de lado las demás actividades en lo que concierne a trabajos que se presentan para titulación 0 en investigaciones, la ley debe actuar rápido y eficazmente. Para Betancur (2016) define a la ética como: "la reflexión del propio modelo de vida - acciones, comportamientos, actos- en donde la razón tiene un papel importante en la toma de decisiones para comprender, justificar y argumentar" pág. 110.

Según Espinoza (2020, pág. 414) nos manifiesta que "La deshonestidad académica ocasiona la devaluación de la obra producto del hecho deshonesto e invalidación de los títulos, certificaciones u otros reconocimientos; así como el rechazo de la comunidad científica, el acoso mediático y la pérdida del respeto". El plagio se encuentra presente dentro de las instituciones educativas, es tarea de todos los que forman parte del sistema educativo como: estudiantes, maestros y padres de familia erradicarlo. Pero si bien es cierto viéndolo desde otra perspectiva la realidad es que este problema es abarcado, ya que los usuarios seguirán recurriendo a fuentes no confiables, revistas, páginas web, libros, etc., buscando su facilidad y beneficio del mismo.

Es de vital importancia reconocer que la tecnología tiene mucho que ver con esta acción de plagiar, por ende, la facilidad de acceso a internet permite que el alumno pueda obtener toda información posible. Esto ha hecho que tanto profesionales como estudiantes mayormente busquen formas fáciles de realizar sus tareas con el objetivo de obtener una buena nota sin darse cuenta que la información copiada de la web no tiene una referencia apropiada de fuentes Soto (2012). La LOEI menciona que: "la deshonestidad académica incluye actos de plagio, trampa o fraude y debería ser sancionado a quien lo cometa por lo tanto no se debería dejar pasar por alto este tipo de faltas" Asamblea Nacional de la República del Ecuador (2014, pág., 62).

La flexibilidad en el proceso de enseñanza aprendizaje hace que los estudiantes comentan dicho acto. Para Espinoza (2020, pág. 411) afirma que "en algunos casos el alumno no se ha preparado en metodologías para gestionar la información, evaluar, emplear y citar adecuadamente las fuentes bibliográficas". Esto solo nos deja en claro como una de los efectos que ocasiona el plagio es causa de la inestabilidad de una buena praxis educativa. Analizando que puede ser proveniente de las directrices que se ponen en manifiesto en nuestro currículo.

Al respecto Ramírez (2017) comenta que una de las acciones que se debe considerar primordial para voltearle la cara al plagio académico es encontrar y darse cuenta de los beneficios que generar el realizar bien un trabajo 0 investigación que manden como tarea los docentes. Estamos seguros de que el mérito por poseer una buena redacción, utilización de citas y reconocimiento de autoría conlleva ventajas enriquecedoras. Puesto que el citar de manera correcta expande su conocimiento y puede generar uno nuevo, y con ello nuevas ideas, opiniones y propuestas en torno al tema que se esté investigando dado que si nos 
direccionamos en el ámbito académico conlleva a un éxito.

Por otro lado, Soto (2012) afirma que la deshonestidad académica, es una acción que nace en la redacción de escritos. Si bien es cierto lo que se quiere expresar es que se debe tomar como punto de partida el buen uso de la escritura desde la educación básica, para que en el transcurso de su vida estudiantil se familiaricen con la redacción haciendo uso adecuado de las reglas gramaticales. Es importante como el estudiante se desenvuelve a la hora de leer un texto, la destreza de innovar, el estudio y como llega a obtener una crítica generando opiniones puesto que esto desemboca en el desarrollo mental, desenvolvimiento gestual, creación de textos, entre otros Gantus (2016).

\section{DESARROLLO}

En el transcurso de estos años en nuestra sociedad se observa como aún permiten este tipo de conductas deshonestas en las instituciones educativas. Se le impide al estudiante que desarrolle una esencia de criticidad, para que se conserve su ética o si es conveniente que este la desarrolle. El plagio académico está presente en las aulas y es responsabilidad de todos erradicarlo, unas de las alternativas para lograr este propósito es que desde los años de escolaridad inferiores se fomente el correcto uso de la información, respetando los créditos de quienes ostentan la propiedad intelectual, lo que implica el ejercitarse en citar de manera adecuada y como identificar fuentes confiables para un buen trabajo.

La disposición de información colgada en internet perjudica en un alto nivel actualmente a los estudiantes y se evidencia en los resultados de los egresados que se han visto inmersos en este tipo de problemas, como consecuencia de ello se puede manifestar la existencia de un vacío intelectual, puesto que se encuentran en un círculo de copiar y pegar los datos que se pueden obtener mediante cualquier navegador sin citar de manera correcta (Guamán, Herrera \& Espinoza (2020). Las causas del plagio se las pueden determinar desde varios puntos de vista, pero las a lo largo del manuscrito de abordarán desde la perspectiva axiológica, curricular y tecnológica.
Empezando por el axiológico, este es imperativo puesto que la educación en valores no es solo una tarea de la comunidad educativa sino en conjunto de la institución y de los padres de familia, por ende, la acción que realizan los maestros es guiar y reforzar valores aprendidos en sus hogares, entonces así se podrá desenvolver una mejor conciencia a la hora de realizar deberes. Por otro lado, al revisar el currículo ecuatoriano se ha podido notar que no está orientando el proceso formativo de la investigación para los dicentes. Sin embargo se puede observar que en el perfil de salida consta expresamente que el estudiante actuará con ética al realizar su investigación.

Analizaremos que una buena educación hace que el alumnado pueda tomar decisiones correctas (Burgo et al., 2019). El plagio en nuestro país es muy evidente y posee cifras muy elevadas tiene varias causas, pero así mismo cada una tiene su punto de inicio. La LOEI expresa que: "La deshonestidad académica incluye actos de plagio, trampa, o fraude en el ámbito académico, ya sea con trabajos realizados en la institución educativa como los realizados fuera de ella" Asamblea Nacional de la República del Ecuador (2012, art. 224). El argumento tecnológico en el que hacemos énfasis por la facilidad de acceso a la información debido a que se puede manipular y está a la orden de todo usuario en internet.

\section{Axiológico}

En un artículo de Ochoa y Cueva (2016, pág. 35) confirman que algunos estudiantes no plagian por cuestiones morales expresan: "pues sí, en el colegio uno bajaba las reseñas del rincón del vago. En la universidad me siento mal, ya es una cosa ética conmigo mismo, yo prefiero no presentar nada a presentar algo que no es mío". Cuestionando así un poco sus decisiones por un temor a malas repercusiones, cabe destacar que el simple hecho de dudar pone en manifiesto que el estudiante tiene la necesidad de ejecutar una acción de manera reflexiva.

Una de las principales causas del plagio académico es la reducida formación en valores. Según Uscátegui (2018) afirma que se puede prevenir el plagio educando desde inicios a los estudiantes con normas de ética, el uso adecuado de editar y escritura de trabajo presentados siempre teniendo presente ética en cada accionar. Las buenas prácticas y la guía adecuada de los docentes generarán un buen 
uso de fuentes, a falta de esto el educando no puede tomar una decisión o ejecutar acciones académicas honestas.

Están presentes en las instituciones educativas códigos de ética en los que se encuentra explícitos como los estudiantes deben tener presente en cada una de sus acciones valores que direccionan a un camino de verdad y justicia. En los porcentajes del siguiente artículo Percepción del plagio académico en estudiantes de odontología que el $68 \%$ de la muestra tienen claro que el plagio académico es un delito, pero es aquí donde debemos dejar en claro que este porcentaje lo afirma mayor parte de la población estudiantil que recién ingresa a la universidad, por consiguiente en el transcurso de su vida estudiantil este pensamiento se va desvaneciendo ¿por qué creen que pase esto? quizás porque los valores me exigen ser responsable en mis acciones Castro et al., (2018).

Aseguramos que es pertinente el uso de valores en vínculo con la actividad escolar para el niño, niña o adolescente para que no comentan plagio y así mismo consideramos que los valores que integran como requisito la honestidad son los más relevantes para abordar esta temática. En cuanto Sureda (2015, pág. 109) expresa que "La falta de valores hace que el ser o estudiante en este caso en centros educativos fomenten y promuevan el desarrollo de conductas académicamente honestas y éticamente re levantes".

\section{Curricular}

El currículo según el MINEDUC dice que "es la expresión del proyecto educativo que los integrantes de un país elaboran para promover el desarrollo y la socialización de las nuevas generaciones $y$ en general a todos sus miembros" Currículo (2016). Se determina que se puede visualizar guías donde el docente sabrá qué acciones realizar para completar cada tema de aprendizaje y evidenciar si se logran. Cuando se realiza una lectura de este documento se puede observar como existe generalización en varios de sus puntos y se limita a acciones que no se desarrollan en su totalidad.

Se presenta en la asignatura optativa redacción creativa donde se expone que su eje temático 1 denominado fundamento teórico para la producción creativa en básicos imprescindibles expresa: "Indagar bases de datos digitales y otros recursos de la web con capacidad para seleccionar fuentes según el propósito de lectura y valorar la confiabilidad e interés o punto de vista de las fuentes escogidas", la praxis de redacción, parafraseo, buen uso de fuentes, entre otras acciones que evitan el plagio académico no se encuentran presentes a cabalidad en este documento y como tal no se puede evidenciar el buen uso de fuentes y la eliminación del plagio por parte del alumnado (Curriculo, 2016).

Somos conscientes de que muchos estudiantes caen en la trampa de realizar actos desmedidos por la dificultad como ellos lo denominan que se les atraviesa en su vida escolar. En las pruebas PISA 2018 el 50\% de los estudiantes en Ecuador mostró un margen de desempeño inferior al nivel 2 en lectura. A esto argumentamos que existen ocasiones de copia de fuentes externas sin brindarle el reconocimiento al autor porque sencillamente no poseemos la capacidad de sacar nuestras propias ideas en base al texto ya leído generando así que el plagio académico se aumente de manera desmedida PISA (2018).

Concordamos que podría haber la oportunidad de vincularlo con lo axiológico porque en base a la formación en valores y acción conjunta podremos tener un estudiante que se enfrente a problemáticas cotidianas de deshonestidad académica dando como resultado acciones buenas y una capacidad de ejecutar trabajos de diferente índole de manera correcta, integrando así a su interior una nueva capacidad de tomar decisiones que le servirán a futuro. $Y$ es que más allá de esto está presente que la fusión de ambos concreta un aprendizaje constructivista como lo comenta Díaz Barriga (2006, pág. 10) expone que "El mérito de la perspectiva constructivista es abrir nuevamente, en el campo de la teoría curricular actual, el debate de la formación en valores".

\section{Tecnológico}

Ctrl C - Ctrl V Muchas veces hemos recurrido a estas combinaciones en nuestro teclado, pues se nos es más fácil y nos salvan de algunos aprietos. Como estudiantes lo hemos usado un sin números de veces, pero ¿somos conscientes de las consecuencias que ocasionan el uso excesivo de esto? las causas del plagio y la falta del uso de documentación adecuada es generada por razones que impactan actualmente ejecutadas con mayor frecuencia en el mundo digital, el punto de vista tecnológico el cual hace que los docentes frente a situaciones tan cotidianas como el copia y pega deba tener competencias exorbitantes. 
La deshonestidad académica está a la orden del día dentro de muchas instituciones educativas. Según Diez (2015, pág. 3) en su artículo sostiene "es cualquier comportamiento intencional que busque un beneficio académico, económico, afectivo o de cualquier otra índole que vaya en contra de los principios éticos de las instituciones educativas $y$, en consecuencia, de la sociedad". Tenemos ejemplos de funcionarios públicos de Ecuador que demuestran cómo el plagio académico se da de manera desmedida y muchas veces no se los penaliza como se debe.

Tomando como referencia este caso, podemos abordar el punto tecnológico como una de las causas más importante en cuanto a la facilidad de acceso a la información que posee un individuo en la actualidad. En internet existen muchas páginas, sitios, blog entre otros que brindan información de diversos temas que, si bien en cierto nos ayuda en gran parte a la construcción de una sociedad del conocimiento debido a la cantidad de intercambio de información de estudios e investigaciones, también puede ser un arma de doble filo puesto que están presentes el uso de detección de plagio para editar y así no muestre un porcentaje elevado.

En un trabajo efectuado por Zapata (2019) muestra en su tabla 4 denominada "La percepción de los estudiantes y docentes frente al tema del fraude escolar", como el $94 \%$ de los estudiantes afirma haber copiado y en otro ítem se evidencia que el $71 \%$ expresa no referenciar a los autores empleados, mucho menos elaborar bibliografías, por lo tanto, no otorgan el reconocimiento a quien corresponde. Se requiere un accionar inmediato frente a este tipo de situaciones que estamos seguros acontecen en muchas escuelas y se presencia como el alumnado ejecuta deshonestidades académicas constantemente.

En lo que concierne a estudiantes y su vida escolar se encontrarán dentro de un ámbito de educación superior donde se espera que estos desarrollen sus actividades pertinentes a su grado de educación y si este supera las expectativas seguir guiando al alumnado es fundamental la enseñanza de elaboración de citas. Para Cevallos et al., (2016) nos muestra en la tabla 7 nombrada Frecuencia de utilización de citas bibliográficas en la elaboración de trabajos académicos 187 estudiantes correspondiente al $93.5 \%$ respondieron que no hacen uso de citas bibliográficas en su trabajo de investigación académica datos recogido de estudiantes de la Universidad de Guayaquil.

La mayoría de sitios web actualmente no cuentan con los derechos de autor y son copiadas de forma literal, lo que hace que haya algunas publicaciones con la misma información pero eso en si no es el problema, el problema se da cuando no sabemos discernir bien la información que estamos buscando, para algunas personas es más fácil abrir el primer resultado de su búsqueda y tomarlo como referencia cuando en realidad no es una información adecuada y verificada, no todo lo que se comparte es una buena información la cual puede servir, esta cantidad de información a la que tenemos acceso nos genera algunas dificultades a la hora de seleccionar.

En un estudio de Días et al. (2013) Sobre la frecuencia del comportamiento respecto de plagio, en el ítem de copiar una pequeña sección de un libro o sitio de internet sin citar o referirse al autor se evidencio como el $91.1 \%$ de los estudiantes si lo ha hecho, así mismo, en la sección de copiar gran parte de un libro o sitio de internet sin citar o mencionar el autor solo el $20.1 \%$ dijo que no lo había hecho. Dejando así en claro que la tecnología puede pasar por encima de cualquier varilla de limitación y dirigiéndose así hacia el alcance del plagio académico, tenemos que exigir medidas que sean pertinentes a solucionar este tipo de actos.

En definitiva, todo accionar negativo de un estudiante viene desde su hogar y está relacionado con sus valores éticos-morales, si no se corrige eso a tiempo, poco a poco se volverá costumbre y la enseñanza del docente será en vano. Es por eso, que desde pequeños se debe instruir una cultura positiva, activa y honesta, que sean responsables de sus actos y hagan acciones que ayuden a crecer su intelecto más no de neutralizarlo. En la etapa escolar, tanto los padres de familia como los docentes son pieza clave para orientar al niño por el camino correcto. Con esto, el infante empleará valores personales que le ayuden a actuar positivamente dentro de sus actividades escolares y del hogar.

\section{CONCLUSIONES}

El plagio es una actitud con pocos valores en cualquier ámbito que se presente recurriendo 
con mayor fuerza en las instituciones educativas, con la aparición de las TIC's y el fácil acceso a los documentos virtuales ha hecho que emerja con mayor frecuencia el plagio académico, es así como lo afirma Alfaro y De Juan (2014) quien sostiene que esta práctica se va extendiendo y la Ilaman ciberplagio, el cual comprende en la presentación de trabajos copiados parcial o en su totalidad sin mencionar al autor, este accionar es cuestionado por muchos debido al mal uso del internet.

Se presenta una metodología con bagajes en los procesos de enseñanza-aprendizaje que en efecto desfavorece las buenas prácticas académicas. Consideramos que las causas expuestas conllevan a muchos errores de pensamientos y acciones, para Hernández et al., (2015) opinan que el uso de herramientas tecnológicas se considera como viejas prácticas pero con nuevos medios, el copia y pega que cada vez se vuelve más evidente a la hora de entregar un trabajo, lo que incide en la poca reflexión, falta de ideas y de argumentación en cada uno de los estudiantes de tal manera que esto repercutirá en su formación académica y desenvolvimiento en el transcurso de su vida posterior.

Consideramos pertinente meditación y la adquisición de conciencia para una verdadera formación académica, la cual implica el uso adecuado de fuentes para guiar nuestros conocimientos más no para apropiarnos de ellos ya que las repercusiones podrían afectar en el proceso de preparación educativa, por lo tanto, es necesario el debido esmero a cada una de las indicaciones transmitidas por los instructivos para evitar tantos inconvenientes más la guía adecuada de los docentes.

Dentro del campo académico, el plagio constituye un acto negativo que realizan muchos estudiantes cuando presentan sus tareas, consultas, etc., por tanto, la calificación refleja la honestidad de dicho estudiante. Cabe recalcar, que cada docente tiene la responsabilidad de verificar y detectar si los trabajos que presentan sus alumnos son originales 0 son plagiados, para luego orientarlos a que ese tipo de actos no son de un buen estudiante. Los valores y la ética deben ser parte de la vida diaria del ser humano y más cuando están en el proceso de aprendizaje, es donde deben de tener presente que analizar una información conlleva a generar mejor conocimiento que realizar el copia y pega, que no incide en nada en las diversas capacidades.
El avance tecnológico, ha tenido sus pro's y sus contra's, principalmente el internet, dado que no todas personas lo utilizan para algo productivo sino para malgastar su tiempo desarrollando actividades que no inciden en su aprendizaje diario, entre ellos se encuentra el poco interés que demuestran al realizar un trabajo investigativo, empleando la modalidad de copiar el escrito de otros autores para luego pegar en sus tareas y engañar al docente que él lo ha realizado. Dichos procedimientos van en contra de los valores de cada estudiante, porque deben ser conscientes que esa actitud no deben tomarla, a su vez, deben ser responsable con cada una de las tareas que envía el respetivo docente.

Las definiciones que conllevan autoría se deben respetar, las ideas y pensamientos que se encuentren presentes en el texto deben ser citadas correctamente como norma. Con el pasar del tiempo, el motivo más tradicional para cometer este tipo de delitos es el factor tiempo, ya que el estudiante por presentar a tiempo sus tareas emplea este método de copiar textualmente y no hacerlo propiamente. Además, existen estudiantes que a pesar que lo hacen una vez y lo descubren, vuelven a hacerlo sin temor a ser sancionados y esto se debe a la falta de ética personal dentro de su ser.

La práctica principal que todo estudiante debe tener presente es el amor por la lectura, dado que es la mejor herramienta para eliminar el plagio en todo momento, porque una persona que se dedica a leer, comprender y reflexionar sobre el texto que buscaba, va a desarrollar buenas actitudes y desempeño académico y, por ende, no necesitará plagiar ningún documento al momento de desarrollar sus tareas e investigaciones respectivas. Dentro del currículo académico, las directrices son claras, es decir, toda asignatura tiene sus objetivos de alcance y para ello, el actuar positivamente ayuda a que el aprendizaje se cohesione con la enseñanza y juntos se construya una educación de calidad.

Hay que tener presente, que al realizar algún trabajo es importante utilizar fuentes confiables, académicas y que contengan información valiosa, para así, poderla entender y emitir opiniones o análisis significativos que solventen cada tarea de una forma netamente académica. En definitiva, todo accionar negativo de un estudiante viene desde su hogar y está relacionado con sus valores éticos-morales, si no se corrige eso a tiempo, poco a poco se 
volverá costumbre y la enseñanza del docente será en vano.

Es por eso, que desde pequeños se debe instruir una cultura positiva, activa y honesta, que sean responsables de sus actos y hagan acciones que ayuden a crecer su intelecto más no de neutralizarlo. En la etapa escolar, tanto los padres de familia como los docentes son pieza clave para orientar al niño por el camino correcto. Con esto, el infante empleará valores personales que le ayuden a actuar positivamente dentro de sus actividades escolares y del hogar.

\section{REFERENCIAS BIBLIOGRÁFICAS}

Alfaro Torres, P., \& De Juan Juarez, T. (2014). El plagio académico formar en competencias y buenas prácticas universitarias. Revista de Unidades de Informacion(6), 1-20. Obtenido de https://dialnet.unirioja.es/servlet/artic ulo? codigo $=4909629$

Betancur Jiménez, G. E. (2016). La ética y la moral: paradojas del ser humano. CES Psicologia, 9(1), 109-121. Obtenido de http://www.scielo.org.co/pdf/cesp/v9n 1/v9n1a08.pdf

Burgo Bencomo, O. B., León González, J. L., Cáceres Mesa, M. L., Pérez Maya, C. J., \& Espinoza Freire, E. E. (2019). Algunas reflexiones sobre investigación e intervención educativa. Revista Cubana de Medicina Militar, 48.

Castro , Y., Yoplac, B., Carpio , A., Cosar, J., \& Sihuay, K. (2018). Percepción del plagio académico en estudiantes de Odontología. Educacion Medica, 19(3), 141-145. Obtenido de https://www.sciencedirect.com/science /article/pii/S1575181317300487

Cevallos Torres, L., Guijarro Rodríguez, A., \& López Domínguez Rivas, L. (2016). El mal uso de la información en trabajos de investigación científica. Didactica $y$ Educacion, 7(4), 57-74.

Curriculo. (2016). Ministerio de Educacion. Obtenido de Documentacion: https://educacion.gob.ec/asignaturasoptativas/

Dias, P., Bastos, S., Gandra, M., \& Diaz, J. (2013). Genius, ¿plagio o creatividad? aportes para una discusión sobre las prácticas pedagógicas. Bordon, 65(3).

Diaz Barriga, A. (2006). La educación en valores: Avatares del currículum formal, oculto y los temas transversales. Revista electronica de investigacion educativa, 8(1), 1-15. Obtenido de http://www.scielo.org.mx/scielo.php?sc ript $=$ sci_arttext\&pid $=$ S160740412006000100001

Diez Martinez, E. (2015). Deshonestidad académica de alumnos y profesores. Su contribución en la desvinculación moral y corrupción social. Sinectica(44), 1-17. Obtenido de http://www.scielo.org.mx/scielo.php?sc ript $=$ sci_arttext\&pid $=$ S1665$109 \times 2015000100014$

Ecuador. Asamblea Nacional de la República (2014). Ley Organica de Educacion Intercultural. Reglamento de la LOEI, 1116. Ecuador. Obtenido de https://educacion.gob.ec/wpcontent/uploads/downloads/2017/02/R eglamento-General-a-la-Ley-OrgAnicade-Educacion-Intercultural.pdf

Espinoza Freire, E. (2020). El plagio. El plagio un flagelo en el ambito academico ecuatoriano. Universidad y Sociedad, 12(3), 407-415. Obtenido de http://scielo.sld.cu/pdf/rus/v12n3/221 8-3620-rus-12-03-407.pdf

Gantus, F. (2016). Conocimientos colectivos, obras particulares. Algunas reflexiones en torno al plagio. Perfiles educativos, 38(154), 12-19. Obtenido de 
Sociedad \& Tecnología | Revista del Instituto Tecnológico Superior Jubones | ISSN:

http://www.scielo.org.mx/scielo.php?sc ript $=$ sci_arttext\&pid $=$ S018526982016000400017.

Guamán Gómez, V. J., Herrera Martínez, L., \& Espinoza, E. (2020). Las competencias investigativas como imperativo para la formación de conocimientos en la universidad actual. Conrado, 16(72), 83-88.

Hernández Islas, M., Ponce Rojo, A., Ortiz Lefort, V., \& Vergara Fregoso, M. (2015). Estrategias docentes emergentes para prevenir el plagio académico en alumnos de pregrado. un estudio fenomenográfico con profesores experimentados. CONSENSUS, 20(2), 41-62. Obtenido de http://revistas.unife.edu.pe/index.php/ consensus/article/view/406/363

Ochoa, L., \& Cueva, A. (2016). Percepciones de estudiantes acerca del plagio: datos cualitativos. Encuentros, 14(2), 25-41. Obtenido de http://www.scielo.org.co/pdf/encu/v14 n2/1692-5858-encu-14-02-00025.pdf

PISA. (2018). Instituto Nacional de Evaluacion Educativa. Obtenido de Programa para la Evaluación Internacional de Estudiantes para el Desarrollo: https://goo.gl/KR1bd4

Ramírez Barreto, A. C. (2017). El plagio académico. Experiencias y algunas ideas para desalentarlo de manera más efectiva. Humanidades y Ciencias de la Conducta(70), 7-22. Obtenido de http://www.cic.cn.umich.mx/cn/article/ view/355/191

Soto Rodriguez, A. (2012). El plagio y su impacto a nivel académico y profesional. Revista e-Ciencias de la Información, 2(1), 1-13. Obtenido de https://www.redalyc.org/pdf/4768/476 848735003.pdf
Sureda-Negre, J., Comas-Forgas, R., \& OliverTrobat, M. (2015). Plagio academico entre estudiantes de secundaria y preparatoria: diferencias de genero y procrastinacion. Educomunicacion, 21(44), 103-111.

Uscátegui Peñuela, R. M. (2018). Plagio, ¿falta de ética o desconocimiento? Perspectivas en Nutricion Humana, 20(1), 9-11. Obtenido de http://www.scielo.org.co/scielo.php?pid $=\mathrm{S} 0124-$

41082018000100009\&script=sci_arttex t\&tIng=en

Zapata Padilla, M. (2019). Incidencia ética del fraude académico en los procesos formativos para los estudiantes de undecimo grado en la sede a de la institucion educativa el castillo. Obtenido de Universidad Santo Tomas : https://repository.usta.edu.co/bitstrea $\mathrm{m} /$ handle/11634/21165/2019marcelaz apata. pdf? sequence $=5$ \&isAllowed $=y$ 